\title{
Single Bunch Transverse Intensity Limitations in the SPS
}

\author{
H. Burkhardt
}

\author{
CERN, Geneva, Switzerland
}

\begin{abstract}
Single bunch intensities of $1.15 \times 10^{11}$ protons will be required for the nominal, and $1.7 \times 10^{11}$ protons for ultimate beams in the LHC. These intensities are at the limit of transverse beam stability at injection energy into the SPS and can lead to very fast transverse instabilities that have recently been observed. There has been a major effort to reduce the SPS impedance. We describe and review the results of transverse beam measurements performed in the SPS. This includes transverse impedance measurements based on coherent tune shifts and measurements of growth and decay rates of several head tail modes as function of chromaticity. We also comment on the effect of the flat chamber geometry.
\end{abstract}

Keywords: Collective Instabilities, Impedance PACS: $29.27 . \mathrm{Bd}$

\section{INTRODUCTION}

There has been a substantial effort to reduce the SPS impedance as injector for the LHC, with the shielding of 1200 bellows and pumping ports. The first $20 \%$ of this work was done in the shutdown between the years 1999 and 2000 and the remaining $80 \%$ between 2000 and 2001 [1]. The improvement was nicely confirmed by longitudinal and transverse measurements. A decrease by a factor of about 2.5 was seen in the longitudinal plane [2]. The threshold for longitudinal instabilities (microwave instability) increased accordingly. The transverse impedance was also reduced, by about $\sim 40 \%$ [3]. This smaller, but still significant improvement in the transverse impedance has nearly been cancelled by the installation of five extraction kickers in the shutdown between the years 2002 and 2003. A fast transverse single bunch instability has recently been observed for the first time with protons in the SPS [4, 5, 6], see also Fig. 1. Four more kickers are required for the fast extraction of the SPS beams into the LHC. Their installation is scheduled for the 2005 to 2006 shutdown.

We review measurements and observations related to the transverse impedance.

\section{BEAM BASED IMPEDANCE MEASUREMENTS}

A description of the principles of impedance measurements based on coherent tune shift and growth and decay rates can be found in the work of Sacherer et al. [7].

The effect on the betatron motion of the bunch centroid is a complex frequency shift of

$$
\Delta \omega_{\beta}=\frac{N e c}{4 \sqrt{\pi} \omega_{\beta}(E / e) T_{0} \sigma_{t}} i\left(Z_{\perp}\right)_{\mathrm{eff}}
$$

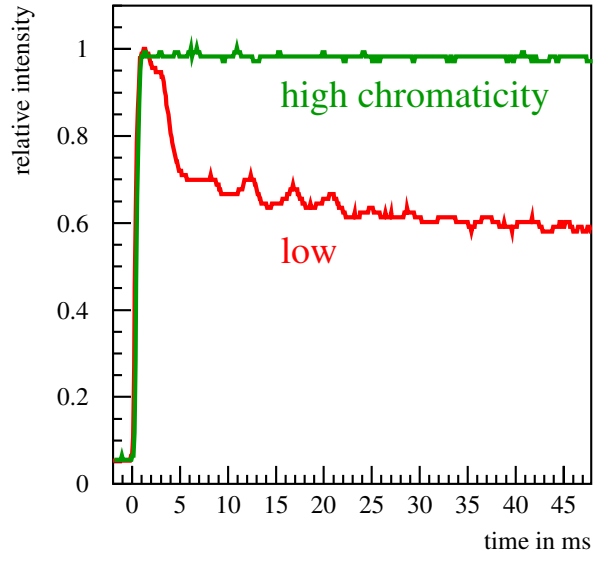

FIGURE 1. Observed bunch intensities following the injection of initially $1.2 \times 10^{11}$ protons. The full bunch length was $4 \sigma_{t}=2.7 \mathrm{~ns}$ and the longitudinal emittance $\varepsilon_{l}=0.2 \mathrm{eVs}$. The fast $(<5 \mathrm{~ms})$ loss of about $30 \%$ of the beam observed at low chromaticity, disappeared when the vertical chromaticity was raised to $\xi_{y}=0.8$.

TABLE 1. Relevant SPS parameters

\begin{tabular}{ccc}
\hline variable & symbol & value \\
momentum & $p$ & $26 \mathrm{GeV} / \mathrm{c}$ \\
revolution frequency & $f_{\text {rev }}$ & $43347.3 \mathrm{~Hz}$ \\
betatron tunes & $Q_{x, y}$ & $\sim 26.2$ \\
synchrotron tune (at $3 \mathrm{MV})$ & $Q_{s}$ & $6.9 \times 10^{-3}$ \\
momentum compaction & $\alpha_{c}$ & $1.919 \times 10^{-3}$ \\
bunch population & $N$ & $0.5-10 \times 10^{10}$ \\
rms bunch length & $\sigma_{t}$ & $\sim 0.5 \mathrm{~ns}$ \\
\hline
\end{tabular}

where $N$ is the number of particles in the bunch, $E$ its energy, $T_{0}$ the revolution frequency, $\sigma_{t}=\sigma_{z} / c$ the bunch length and $\omega_{\beta}=2 \pi Q f_{\text {rev }}$ the betatron frequency. Eq. (1) applies to both $x$ and $y$. A comparison of measurements in the vertical plane is shown in Fig. 2. 


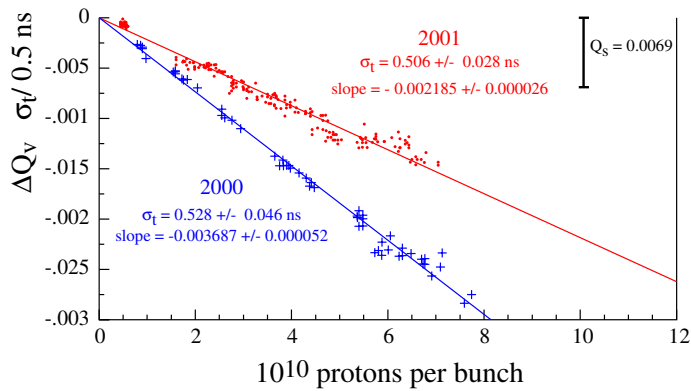

FIGURE 2. Comparison of the measured coherent tune shifts (normalised to the measured bunch length) with intensity in the vertical plane in the years 2000 (before) and 2001 (after the main part of the impedance reduction).

For SPS parameters see Table $1 .\left(Z_{\perp}\right)_{\text {eff }}$ is the effective transverse impedance, which for a round chamber is the impedance convoluted with a weight function $h$ representing the longitudinal bunch shape

$$
\left(Z_{\perp}\right)_{\mathrm{eff}}\left(\omega_{\xi}\right)=\int_{-\infty}^{\infty} Z_{1}^{\perp}(\omega) h_{m}\left(\omega-\omega_{\xi}\right) d w .
$$

For the 0-mode coherent bunch oscillations analysed here and Gaussian bunches the weight function is

$$
h_{0}(\omega)=\frac{\sigma_{t}}{\sqrt{\pi}} e^{-\left(\omega \sigma_{t}\right)^{2}},
$$

that is, a Gaussian in $\omega$ with an rms width of $1 /\left(\sqrt{2} \sigma_{t}\right)$, or equivalently a Gaussian in frequency $f=\omega /(2 \pi)$ of width $\sigma_{f}=1 /\left(2 \pi \sqrt{2} \sigma_{t}\right)$. For $\sigma_{t}=0.5 \mathrm{~ns}$, the rms width in frequency is $225 \mathrm{MHz}$ and the weight has dropped to $5 \%$ at $f=0.275 / \sigma_{t}=551 \mathrm{MHz}$. The Gaussian weight function in Eq. (2) is centred at $\omega_{\xi}$ which depends on the chromaticity $\xi=Q^{\prime} / Q=\frac{\Delta Q}{Q} / \frac{\Delta p}{p}$ and the phase slip factor $\eta$ according to

$$
\omega_{\xi}=\xi \frac{\omega_{\beta}}{\eta} .
$$

For the SPS at $26 \mathrm{GeV}$, we have numerically $f_{\xi}=$ $\omega_{\xi} /(2 \pi)=\xi \cdot 2.08 \mathrm{GHz}$.

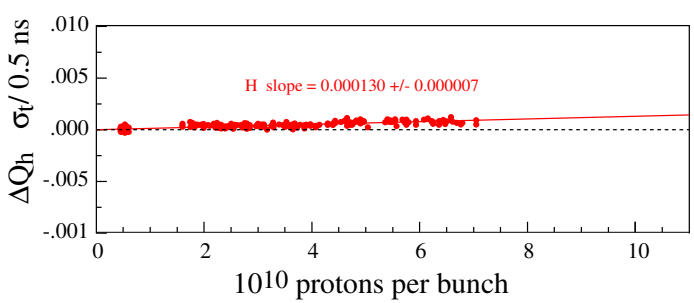

FIGURE 3. Coherent tune shift (normalized to bunch length) with intensity in the horizontal plane recorded in 2001.

The visible coherent tune shift in the horizontal plane with current in the SPS is very small, compatible with zero or even slightly positive, see Fig.3. The SPS has a rather flat vacuum chamber geometry (with $\mathrm{V} / \mathrm{H}$ ratios from $1 / 3$ to $1 / 4$ in the bending sections). The resulting quadrupole wake fields enhance the vertical and cancel the horizontal tune shift with current [8]. For an explanation of a small positive slope in the context of resistive wall theory see [9].

Fig. 4 shows the principle of the determination of growth and decay rates. The growth and decay rates are obtained from the evolution of the tune peak heights of sub-samples.

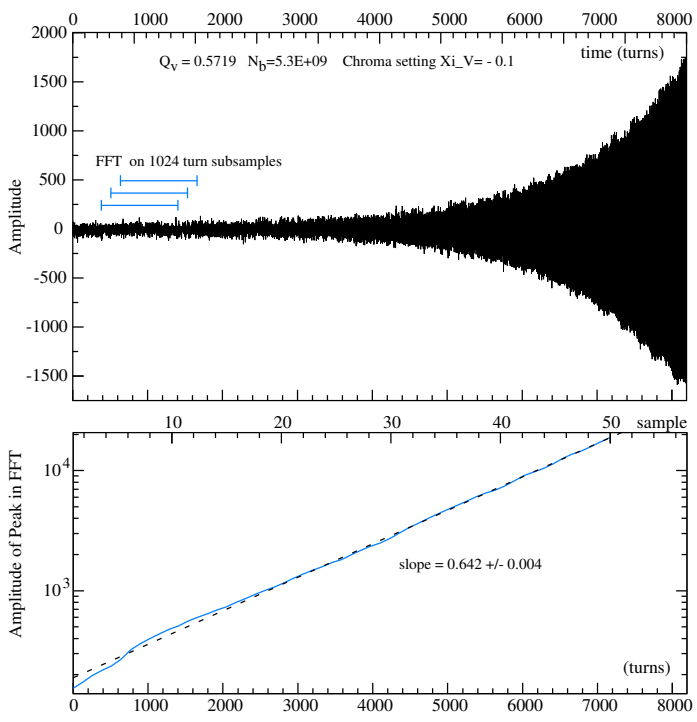

FIGURE 4. Growth/decay determination. The vertical chromaticity was set slightly negative at the start of the measurement. Vertical turn to turn amplitudes recorded with the tune meter on successive turns are shown on the top and the height of the tune peak of sub-samples is displayed with logarithmic $y$-scale in the bottom of the picture. The time constant $\tau$ of the exponential growth rate is obtained from the slope of the straight dashed line.

These measurements are performed over a broad range of chromaticities. The growth and decay rates can be very fast. The measurements are done at low intensity, typically about $5 \times 10^{9}$ to obtain longer observation times (at least several hundred turns). The real part of the (effective) transverse impedance is obtained from these measurements as function of frequency. A result is shown in Fig.5, and compared with a predictions based on a single frequency broad band $(Q=1)$ impedance or the sum of two broad band impedances.

For positive chromaticities, it is possible to distinguish several higher order head-tail modes and to determine their respective damping rates [3]. 


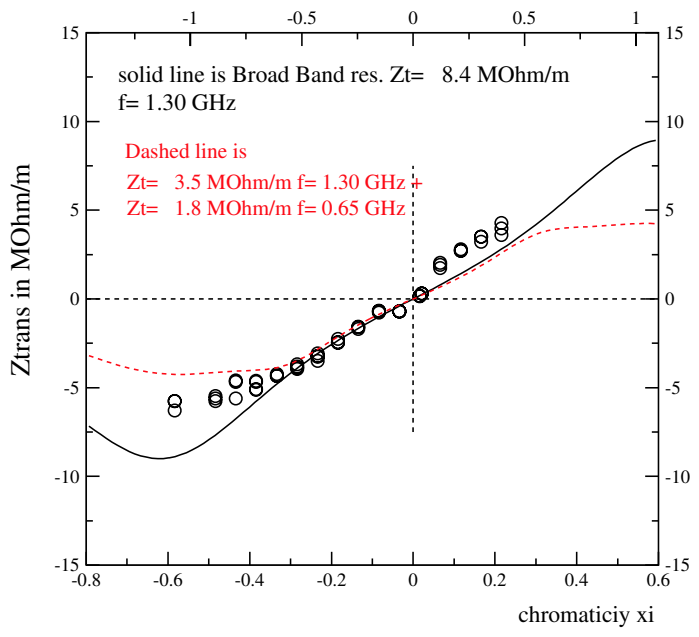

FIGURE 5. Measured $\operatorname{Re} Z_{y}$ (dots) and fits based a one or two broad band impedances.

TABLE 2. $\operatorname{Im} Z_{t}$ in $M \Omega / m$.

\begin{tabular}{ccc} 
Year & Horizontal & Vertical \\
2000 & $-1 \pm 2$ & $\sim 30$ \\
2001 & $-.5 \pm .5$ & 18 \\
2002 & & 15 \\
2003 & & 24 \\
\hline
\end{tabular}

\section{Results in terms of (effective) Impedance}

The results quoted in Tables 2, 3 have been obtained using directly Eq.1, without flat chamber correction. A relative uncertainty of about $\pm 10-20 \%$ is estimated for $\operatorname{Im} Z_{t}$, as relevant for comparison of changes between the years. The absolute uncertainty, relevant for the comparison with simulations is larger, and estimated at about $30 \%$ (taking into account uncertainties in the knowledge of non-perfectly Gaussian longitudinal profiles, a small fraction of uncaptured beam, ..). About a factor of 2 uncertainty is estimated for the knowledge of $\operatorname{Re} Z$ from growth/decay rates. The results of $\operatorname{Re} Z$ apply to a single broad band impedance with a resonance frequency of $f_{r}=1.3 \mathrm{GHz}$.

\section{CONCLUSION}

Following various hardware changes, it is now the transverse impedance which potentially limits single bunch currents in the SPS. The impedance will still increase due to installation of kickers required for the LHC. A further effort to reduce the SPS impedance, this time with emphasis on the transverse plane, should be launched and will likely require new or modified kickers, to be able to increase intensities towards the "ultimate" $1.7 \times 10^{11}$
TABLE 3. $\operatorname{Re} Z_{t}$ in $\mathrm{M} \Omega / \mathrm{m}$.

\begin{tabular}{ccc}
\hline Year & Horizontal & Vertical \\
2001 & 5 & 9 \\
2003 & 7 & 10 \\
\hline
\end{tabular}

protons per bunch planned for the LHC.

\section{ACKNOWLEDGEMENT}

The measurements described here were performed over several years and involved G. Arduini, F. Zimmermann, E. Vogel, M.Zorzano, A. Koschik, Y. Papaphilippou, J. Wenninger and G. Rumolo who in addition contributed a lot with detailed simulations. From the rf-group I would like to thank T. Bohl, R. Baudrgenghien, T. Linnecar and E. Shaposhnikova. Finally, I would like to acknowledge stimulating discussions on predictions and theory with B. Zotter, J. Gareyte, L. Vos and E. Metral.

\section{REFERENCES}

1. P. Collier et al., "Reducing the SPS machine impedance", Prepared for 8th European Particle Accelerator Conference (EPAC 2002), Paris, France, 3-7 Jun 2002.

2. T. Bohl, T. Linnecar, and E. Shaposhnikova, "Impedance reduction in the CERN SPS as seen from longitudinal beam measurements", 8th European Particle Accelerator Conference : a Europhysics Conference, La Vilette, Paris, France, 3-7 Jun 2002.

3. H. Burkhardt, G. Rumolo, and F. Zimmermann, "Coherent beam oscillations and transverse impedance in the SPS", Proc. EPAC 2002 pp. 1449 - 1451 and CERN-SL-2002-030.

4. G. Arduini, H. Burkhardt, and E. Metral, "Observation of a fast single bunch instability on protons in the SPS", CERN AB-Note-2003-093 (MD), Dec. 2003.

5. H. Burkhardt, G. Arduini, E. Benedetto, E. Métral, and G. Rumolo, "Observation of a Fast Single-Bunch Transverse Instability on Protons in the SPS", CERN-AB2004-055 and Proc. EPAC 2004.

6. E. Metral, G. Arduini, et al., "Transverse Mode-Coupling Instability in the CERN Super Proton Synchrotron”, these proceedings.

7. F. Sacherer, "Single-beam collective phenomena, Transverse, Bunched Beams", Proceedings of the 1976 Erice school on accelerators, M.H. Blewett (Ed.), CERN yellow report 77-13, (1977) p. $198 \mathrm{ff}$.

8. J. Gareyte, "Impedances: Measurements and Calculations for Non-symmetric Structures", Proc. EPAC 2002 pp. 89 93 and CERN-SL-2002-028.

9. H. Burkhardt, A. Koschik, G. Rumolo, F. Zimmermann, and B. Zotter, "Coherent tune shifts measured with few bunches in the SPS and comparison with resistive wall theory", Prepared for Particle Accelerator Conference (PAC 03), Portland, Oregon, 12-16 May 2003 and CERN-AB-2003-014-ABP. 